\title{
Los pastos estrella africana, kikuyo y "rye grass" en Cartago, Costa Rica: biomasa, composición botánica y nutrientes
}

\section{Luis Villalobos Villalobos ${ }^{1}$ iD \& Rodolfo WingChing-Jones ${ }^{2}$}

1. Universidad de Costa Rica, Escuela de Zootecnia, Centro de Investigación en Nutrición Animal, San José, Costa Rica; luis.villalobosvillalobos@ucr.ac.cr

2. Universidad de Costa Rica, Escuela de Zootecnia, Centro de Investigación en Nutrición Animal (CINA), San José, Costa Rica; rodolfo.wingching@ucr.ac.cr

Recibido 28-VIII-2019 • Corregido 9-XI-2019 • Aceptado 12-XII-2019

DOI: https://doi.org/10.22458/urj.v12i1.2811

\begin{abstract}
African Stargrass, Kikuyograss and Ryegrass in Cartago, Costa Rica: biomass, field composition and nutrients". Introduction: Forage is the main feedstuff for dairy cattle and its quality and biomass define optimal nutritional balances. Objectives: To evaluate the biomass, botanical composition, and crude protein, neutral detergent fiber and digestibility of Stargrass, Kikuyugrass and perennial Ryegrass with 30,30, and 38 days of regrowth, respectively, located in the foothills of Irazu volcano, Costa Rica. Methods: We collected forage samples in paddocks 1-2 days before grazing from June 2016 through July 2017, 20 samples in each forage. We estimated biomass by using a visual assessment technique and separated each sample in three subsamples for nutritional analyses, number of leaves per tiller, and botanical composition. Results: We found greater allowance (kg DM/cow/paddock), protein, and digestible fiber in Stargrass. We found no significant differences in nutrients between Kikuyu and Ryegrass. We used four groups to classify the plant cover in the paddocks. The main species of grass varied between $50-99 \%$ while other grasses represented $0-36 \%$ of the plant cover. The percent of weeds and legumes were $0-7,5 \%$ and $0-15 \%$ in the paddocks, respectively. We found that senescent material could represent up to $46 \%$ in some of the paddocks. Conclusion: The botanical composition in the paddocks had no effect on the nutritional value of the three grasses.
\end{abstract}

Keywords: Forages, weed, crude protein, digestibility, fiber.
RESUMEN. Introducción: El forraje es el alimento principal para el ganado lechero y su calidad y biomasa definen equilibrios nutricionales óptimos. Objetivo: Valoramos la disponibilidad de biomasa, la composición botánica, los contenidos de proteína cruda, fibra detergente neutro y la digestibilidad del pasto estrella africana, kikuyo y ryegrass bajo pastoreo, ubicados en las faldas del volcán Irazú, Costa Rica con 30, 30 y 38 días de rebrote, respectivamente. Métodos: De junio 2016 a julio 2017, recolectamos muestras de los tres forrajes, 1-2 días antes del ingreso de los animales, para 20 muestreos en cada forraje, mediante el uso de una técnica visual que permite determinar la biomasa disponible en cada aparto. Esta muestra se dividió en tres partes, para el análisis de composición nutricional, número de hojas y composición botánica. Resultados: Encontramos que el pasto estrella africana ofrece mayor cantidad de materia seca disponible (Kg MS/animal/potrero), proteína disponible, FDN digestible y materia seca digestible que los pastos kikuyo y ryegrass. Además, no determinamos diferencias en los aportes nutricionales entre el pasto kikuyo y ryegrass. Identificamos cuatro coberturas en los potreros, donde la gramínea principal fluctuó entre 50 y $99 \%$, la secundaria, entre 0 y $36 \%$, las arvenses y leguminosas de 0 a $7,5 \%$ y 0 a $15 \%$ respectivamente. Mientras que, el material senescente vario entre 0 a $46 \%$ según la especie forrajera. Conclusión: La composición botánica en los potreros no tuvo efecto sobre el valor nutricional de los tres pastos.

Palabras clave: Forraje, malezas, proteína cruda, digestibilidad, fibra. 
El área de cultivo de forraje en Costa Rica equivale al 25\% del territorio nacional (INEC, 2015), área cubierta por gramíneas y leguminosas de piso para el pastoreo de los semovientes y en sistemas de corta y acarreo. Por sus características inherentes, se pueden agrupar por pisos altitudinales, resistencia a enfermedades y plagas (Castillo, 2006), adaptabilidad a suelos con pH bajos (Jiménez, Granados, Oliva, Quiroz, \& Barrón, 2010), alta salinidad (Cardamone, Cuatrín, Grunberg, \& Tomás, 2018) y humedad. Como meta, los sistemas de producción buscan optimizar la relación entre productividad por hectárea del cultivo y aporte efectivo de nutrimentos. Por consiguiente el requerimiento nutricional del semoviente es cubierto por la sumatoria de los aportes del forraje consumido, más lo suministrado en la canoa, como el alimento balanceado, forraje de corta, forrajes conservados, subproductos agrícolas y aditivos nutricionales (NRC, 2001).

Desde la perspectiva de manejo del cultivo, la meta se logra al recolectar información de la producción de biomasa producida y la composición botánica de este material a través del tiempo, para obtener un inventario forrajero, que permita proyectar la capacidad de carga de la finca, como también, las variaciones del aporte nutricional. Estudios de esta índole en Costa Rica, describen la producción y calidad del pasto estrella africana, kikuyo y ryegrass; a diferentes edades de cosecha. En el caso del pasto estrella (28 días/pastoreo), se registran producciones de biomasa de $4642 \mathrm{~kg}$ MS/ha/ciclo en promedio (Salazar, 2007), con porcentajes de aprovechamiento de $43,05 \%$ y contenidos de materia seca (MS\%), proteína cruda (PC\%), fibra detergente neutro (FDN\%), fibra detergente ácida (FDA\%), extracto etéreo (EE\%) y cenizas de 22,09; 20,25; 72,81; 37,88; 0,91 y 8,69\% respectivamente (Salazar, 2007). Para el pasto Kikuyo, se informa de una disponibilidad a 28 días de rotación de $7108 \mathrm{~kg} \mathrm{MS} / \mathrm{ha}$ (Andrade, 2006), con valores porcentuales de MS, PC, FDN, FDA, EE y cenizas de 20,19; 22,62; 58; 29; 3,05 y 9,05\% respectivamente (Andrade, 2006). Por último, en el pasto Ryegras se resume una producción promedio de $4110 \mathrm{~kg} \mathrm{MS} /$ ha/ciclo y valores de MS, PC, FDN, FDA, EE y cenizas de 15,$92 ; 25,21 ; 49,76 ; 25,57 ; 2,61$ y $9,96 \%$ respectivamente (Villalobos, 2006).

Sumado a este ejercicio de caracterizar la productividad del cultivo, valorar su composición botánica y su dinámica según el pastoreo, es una señal de sostenibilidad de la pastura, debido a que las coberturas secundarias, compiten por espacio, radiación, nutrimentos de la solución del suelo con el cultivo principal (Galon et al., 2018), presentan alelopatías que reducen la viabilidad de la pastura (Yuan et al., 2018), albergan poblaciones de nematodos que afectan las raíces del cultivo principal (Núñez, 2017); además de reducir la disponibilidad (Harker, Baron, Chanasyk, Naeth, \& Stevenson, 2000) y la calidad de la materia seca comestible para los semovientes (Belle, Marchi, Martins, Sousa, \& Pinheiro, 2018). En el caso de la composición botánica, los estudios en pasto estrella, kikuyo y ryegrass, describen además de la cobertura principal, la presencia de otras gramíneas, arvenses y material senescente. Valores que fluctúan para la cobertura principal entre 82,56 y 95,11 para Estrella africana (Salazar, 2007), de 77,42 a 86,5 en Kikuyo (Andrade, 2006) y 67,72 a 80,63 en Ryegrass (Villalobos, 2006). Para las otras coberturas analizadas, en el pasto estrella, se informan rangos a través del año para las otras gramíneas, malezas y material senescente de 1,04 a 4,11; de 0 a 5,44 y de 2,72 a 10,93\% respectivamente (Salazar, 2007). Mientras que en el pasto Kikuyo, los rangos fueron de 8,26 a 14,$4 ; 0,43$ a 0,77 y 3,11 a 6,09\% respectivamente (Andrade, 2006). En el caso del pasto ryegrass, los valores descritos fueron de 9,29 a 18,61; de 0,49 a 2,02 y de 0,27 a 6,01, para las coberturas de otras gramíneas, arvenses y material senescente respectivamente (Villalobos, 2006). El conocer la composición de la pastura es una herramienta de medición que permite categorizar el grado de degradación que presenta el cultivo, donde valores mayores a $10 \%$ de malezas, $2 \%$ de leguminosas y menos del $80 \%$ de la biomasa proveniente de la gramínea principal, son indicadores del inicio de un proceso de degradación (Garzón \& Mora, 2014).

Por tal motivo, el objetivo de esta propuesta fue valorar la disponibilidad de biomasa, la composición botánica, los contenidos de proteína cruda, fibra detergente neutro y la digestibilidad 
del cultivo del pasto estrella africana, kikuyo y ryegrass bajo pastoreo, ubicados en las faldas del volcán Irazú, Costa Rica.

\section{MATERIALES Y MÉTODOS}

Ubicación de los sistemas: Los tres forrajes utilizados en esta investigación se ubicaron en la provincia de Cartago, Costa Rica, en las faldas del Volcán Irazú. En el caso del pasto estrella

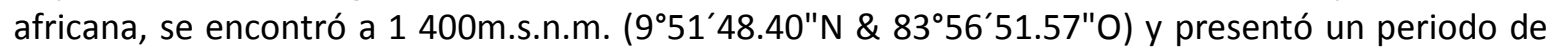
descanso/ocupación de 30/1 días. Para el pasto Kikuyo, el periodo de descanso/ocupación fue de $30 / 0,5$ días, a una altura de 2400 m.s.n.m. (9 $\left.56^{\prime} 24.90^{\prime \prime} \mathrm{N} \& 83^{\circ} 52^{\prime} 44.28^{\prime \prime O}\right)$. Por último, el pasto Ryegrass, presento un ciclo de descanso/ocupación de 38/0,5 días y se ubicó a 2800 m.s.n.m. (9 $\left.57^{\prime} 25.39^{\prime \prime} \mathrm{N} \& 83^{\circ} 49^{\prime} 57.21^{\prime \prime} \mathrm{O}\right)$

Periodo experimental: De junio 2016 a julio 2017, muestreamos los tres forrajes, entre uno o dos días antes del ingreso de los animales, para un total de 20 muestreos en cada forraje durante el periodo experimental. Este muestreo consistió en la utilización de una técnica visual (Botanal $\left.{ }^{\circledR}\right)$ para la determinación de la biomasa disponible en cada aparto (Hargraves \& Kerr, 1978). Esta asocia un valor numérico $(1,2$ y 3 ) a una cobertura característica dentro del potrero, donde se considera la altura y la densidad del forraje; el valor de uno considera un estrato con baja disponibilidad, dos, con una disponibilidad media y tres con una alta disponibilidad de forraje. En una primera etapa, el aparto se inspecciona para definir y ubicar los tres estratos, posterior a esta identificación, el aparto se recorre de forma sistemática, donde cada 5 pasos, se determina el tipo de estrato al frente de la persona que aplica el muestreo. Las lecturas se realizan hasta completar un mínimo de 60 puntos. Al finalizar, con ayuda de un marco de $0,50 \mathrm{~m}$ de lado, se cosecha en bolsas individuales los estratos $(1,2$ y 3$)$ definidos en la primera etapa de esta técnica, a una altura de $10 \mathrm{~cm}$. Estas tres muestras se pesan y se multiplica cada una con su respectiva frecuencia relativa, tomando como base las 60 muestras visuales. Estos productos (peso estrato $(1,2,3)$ * frecuencia relativa $(1,2,3)$ se suman y se dividen entre el total de muestras visuales tomadas, para obtener la ponderación de la biomasa disponible por aparto en $0,25 \mathrm{~m}^{2}$. En el caso de este trabajo, este valor se multiplicó por el tamaño del aparto y se dividió entre el número de animales, para obtener los kilogramos de biomasa disponible promedio por animal en cada aparto.

De forma proporcional a los resultados obtenidos por la técnica visual de muestreo en cada forraje, se tomó una porción del forraje de forma aleatoria de cada estrato, para obtener una muestra compuesta, la cual fue enviada al laboratorio de bromatología del Centro de Investigación en Nutrición Animal (CINA) de la Universidad de Costa Rica, donde se realizaron los análisis de materia seca (MS), proteína cruda (PC), fibra detergente neutro (FDN), digestibilidad de la fibra detergente neutro (dFDN) y digestibilidad de la materia seca (DIVMS) (AOAC,1991).

En el caso de la determinación del número de hojas uno o dos días antes del pastoreo, en cada muestreo se realizaron 50 observaciones aleatorias, con la finalidad de contar el número de hojas, para tal fin, solo se consideraron las hojas verdes, descartando las hojas senescentes en cada rebrote. Al obtener estas 50 observaciones, los valores obtenidos se promediaron, para obtener el número de hojas promedio al momento del pastoreo.

Por último, para la determinación de la composición botánica de la pastura, se utilizó, la plantilla del programa Botanal ${ }^{\circledR}$, la cual asume y clasifica las tres principales especies en orden descendente, en cobertura mayor $(70,2 \%)$, media $(21,1 \%)$ y baja $(8,7 \%)$. Permitiendo repetir una especie dos o tres veces en la categorización, tal como ocurrió en los potreros de este estudio con los tres pastos que se consideran monocultivos. Partiendo de este supuesto, en cada lectura se realizaba una separación visual de las coberturas presentes, valor que se ponderó entre el número de muestras visuales, para cada cobertura evaluada. 
Análisis de la información: La información de cada potrero se organizó en un diseño irrestricto al azar, para definir el efecto de la composición botánica del aparto (proporción gramínea principal, gramínea secundaria, arvenses, leguminosas y material senescente) sobre la disponibilidad de biomasa, el contenido de proteína cruda, fibra detergente neutro y la digestibilidad de la materia seca y de la fibra. De igual manera, con ayuda del comando Proc GLM del programa SAS (2011), se comparó los indicadores de producción de biomasa, número de hojas, contenido de proteína, fibra y la digestibilidad de los tres forrajes analizados.

Ética, conflicto de interés y declaración de financiamiento: Los autores declaramos haber cumplido con todos los requisitos éticos y legales pertinentes, tanto durante el estudio como en el manuscrito; que no hay conflictos de interés de ningún tipo, y que todas las fuentes financieras se detallan plena y claramente en la sección de agradecimientos. Asimismo, estamos de acuerdo con la versión editada final del documento. El respectivo documento legal firmado se encuentra en los archivos de la revista.

\section{RESULTADOS}

Productividad y aporte nutricional: Al separar las especies forrajeras estudiadas según la altitud del sistema productivo, se nota que la especie cultivada por debajo de los $2000 \mathrm{~m} . \mathrm{s} . \mathrm{n} . \mathrm{m}$, supera en 2,68 y 2,40 veces los kg MS/animal/potrero disponible para cada animal en el caso del pasto kikuyo y Ryegrass respectivamente (Cuadro 1 ). De igual manera, al considerar el número de hojas de los materiales forrajeros al momento de la cosecha, el pasto estrella africana, presenta 1,64 y 2,70 más hojas, que los pastos cultivados a 2400 y 2800 m.s.n.m., respectivamente. En el caso de los kilogramos de proteína disponible para los animales durante el pastoreo, en este trabajo se determinó que el pasto estrella (4,56kg PC) aporta 2,28 y 2,21 veces más PC que el pasto kikuyo ( $2 \mathrm{~kg}$ $\mathrm{PC})$ y ryegrass $(2,06 \mathrm{~kg})$ respectivamente. Al evaluar la disponibilidad de la FDN digestible para los semovientes en cada aparto, se encontró que el pasto estrella $(10,24 \mathrm{~kg}$ FDN) supera al pasto kikuyo (3,89 kg FDN) y ryegrass (4,48kg FDN) en 2,63 y 2,28 veces respectivamente. Por último, los kilogramos de materia seca digestible disponible para los animales, fue de $22,18 \mathrm{~kg}$ en el pasto estrella, 9,33kg en el pasto kikuyo y de 10,71kg en el pasto ryegrass, presentando una proporción de 2,38 y 2,07 respectivamente. En el caso de la relación entre el pasto kikuyo y el ryegrass para los kg de PC, FDN digestible y la materia seca digestible disponible en cada aparto fue cercana a uno.

En las tres especies forrajeras estudiadas, la proporción de la cobertura de la gramínea principal, fluctúo entre el 50 y 99\% (Cuadro 2). Para el caso de la cobertura secundaria, está alcanzó un máximo de $35,61 \%$ en el pasto ryegrass, un valor intermedio en el pasto estrella $(19,13 \%$ ) y un valor de 9,22\% en la pastura de kikuyo. Los valores máximos de arvenses obtenidos en este trabajo fluctuaron entre 1,12 hasta 7,52\% (Cuadro 2). Los forrajes por encima de los $2000 \mathrm{~m}$ s.n.m presentaron mayor proporción de plantas leguminosas que en el caso del pasto estrella, mientras que la proporción de material senescente superó el $40 \%$ de la cobertura en las pasturas de estrella y kikuyo, no así en el pasto ryegrass, que obtuvo un valor máximo de $15,2 \%$. Valores de otras coberturas hasta un valor promedio de $18,31,17,32$ y $21,65 \%$ en los forrajes estrella africana, kikuyo y ryegrass, respectivamente; no afectan la cantidad (kg MS) ni la calidad (PC, DIVMS y FDN) de la materia seca disponible para los semovientes al momento del pastoreo (Cuadro 3). 


\section{Cuadro 1}

Producción de biomasa, número de hojas, contenido de proteína, fibra y digestibilidad de la materia seca de estrella africana, kikuyo y Ryegrass bajo pastoreo

\begin{tabular}{|c|c|c|c|c|c|c|}
\hline \multirow{2}{*}{ Variable } & \multicolumn{6}{|c|}{ Especie forrajera } \\
\hline & $\mathbf{N}$ & Estrella Africana & $\mathbf{N}$ & Kikuyo & $\mathbf{N}$ & Ryegrass \\
\hline Biomasa disponible (Kg MS/animal/potrero) & 20 & $29,11^{a}$ & 20 & $10,86^{b}$ & 20 & $12,16^{b}$ \\
\hline Número hojas verdes/rebrote & 20 & $6,56^{a}$ & 20 & $3,99 \mathrm{~b}$ & 20 & $2,43^{c}$ \\
\hline Proteína cruda (\%) & 18 & $15,66^{b}$ & 18 & $18,45^{a}$ & 18 & $16,94 a b$ \\
\hline Fibra detergente neutro (\%) & 20 & $57,43^{a}$ & 20 & $48,89 \mathrm{~b}$ & 20 & $47,80^{b}$ \\
\hline Digestibilidad de la FDN (\%) & 20 & $61,26^{c}$ & 20 & $73,31^{b}$ & 20 & $77,17^{\mathrm{a}}$ \\
\hline DIVMS (\%) & 20 & $76,21^{c}$ & 20 & $85,95^{b}$ & 20 & $88,11^{a}$ \\
\hline
\end{tabular}

* Letras distintas en una misma variable significan diferencias significativas entre especies forrajeras $(p>0,5)$.

\section{Cuadro 2}

Composición botánica de apartos de estrella africana, Kikuyo y ryegrass en pastoreo

\begin{tabular}{lcccc}
\hline \multirow{2}{*}{ Tipo de cobertura } & \multicolumn{4}{c}{ Composición de la cobertura (\%) } \\
\cline { 2 - 5 } & N & Mínimo & Promedio & Máximo \\
\cline { 2 - 5 } & \multicolumn{4}{c}{ Estrella Africana } \\
\hline Gramínea principal & 20 & 53,44 & 81,69 & 99,13 \\
Gramínea secundaria & 20 & 0 & 2,56 & 19,13 \\
Arvenses & 20 & 0 & 0,29 & 1,12 \\
Leguminosas & 20 & 0 & 0,07 & 0,96 \\
Material senescente & 20 & 0 & 15,39 & 46,01 \\
\hline & \multicolumn{4}{c}{ Kikuyo } \\
Gramínea principal & 20 & 54,43 & 83,24 & 99,65 \\
Gramínea secundaria & 20 & 0 & 2,7 & 9,22 \\
Arvenses & 20 & 0 & 1,02 & 4 \\
Leguminosas & 20 & 0 & 1,54 & 5,48 \\
Material senescente & 20 & 0 & 12,06 & 44,03 \\
\hline & \multicolumn{5}{c}{ Ryegrass } \\
Gramínea principal & 20 & 49,4 & 78,33 & 96,01 \\
Gramínea secundaria & 20 & 0 & 10,85 & 35,61 \\
Arvenses & 20 & 0 & 1,52 & 7,52 \\
Leguminosas & 20 & 0 & 6,71 & 14,66 \\
Material senescente & 20 & 0 & 2,57 & 15,2 \\
\hline
\end{tabular}




\section{Cuadro 3}

Significancia del tipo de cobertura sobre la biomasa disponible y la calidad nutricional de la estrella africana, kikuyo y ryegrass en pastoreo

\begin{tabular}{|c|c|c|c|c|}
\hline \multirow{2}{*}{ Tipo de cobertura } & $\begin{array}{c}\text { Biomasa disponible } \\
\text { (Kg MS/animal/potrero) }\end{array}$ & $\begin{array}{c}\text { Proteína cruda } \\
\text { (\%) }\end{array}$ & $\begin{array}{c}\text { Fibra detergente } \\
\text { neutro (\%) }\end{array}$ & DIVMS (\%) \\
\hline & \multicolumn{4}{|c|}{ Estrella africana } \\
\hline Gramínea principal & $n s^{*}$ & ns & ns & ns \\
\hline Gramínea secundaria & ns & ns & ns & ns \\
\hline Arvenses & ns & ns & ns & ns \\
\hline Leguminosas & ns & ns & ns & ns \\
\hline \multirow[t]{2}{*}{ Material senescente } & ns & ns & ns & ns \\
\hline & \multicolumn{4}{|c|}{ Kikuyo } \\
\hline Gramínea principal & ns & ns & ns & ns \\
\hline Gramínea secundaria & ns & ns & ns & ns \\
\hline Arvenses & ns & ns & ns & ns \\
\hline Leguminosas & ns & ns & ns & ns \\
\hline \multirow[t]{2}{*}{ Material senescente } & ns & ns & ns & ns \\
\hline & \multicolumn{4}{|c|}{ Ryegrass } \\
\hline Gramínea principal & ns & ns & ns & ns \\
\hline Gramínea secundaria & ns & ns & ns & ns \\
\hline Arvenses & ns & ns & ns & ns \\
\hline Leguminosas & ns & ns & ns & ns \\
\hline Material senescente & ns & ns & ns & ns \\
\hline
\end{tabular}

${ }^{*}$ ns: no significativo; $p>0,05$

\section{DISCUSIÓN}

Los tres cultivos estudiados presentan un rango de altura que favorece el desarrollo del mismo, en el caso de este estudio esta diferencia fue 1000 y 1 400m.s.n.m. entre el pasto estrella africana en comparación al pasto kikuyo y ryegrass, respectivamente. Estos rangos de altura generan ecosistemas con gradientes de temperatura, radiación, humedad relativa, nubosidad, humedad del suelo, temperatura del suelo y velocidad del viento (Conejo, 2017); factores que afectan el crecimiento del forraje y la velocidad de recuperación después de un periodo de pastoreo por parte del semoviente. Además del componente ambiental para su adaptación, las características propias del cultivo, al tener crecimiento estolonífero (estrella africana), rizomatoso (Kikuyo) y en cepa (Ryegrass), le permite una mayor cantidad de puntos de crecimiento (yemas), hojas remanentes al pastoreo y estructuras de reserva de carbohidratos (estolones y rizomas), con lo que su recuperación es más rápida en el caso de la estrella y el kikuyo, al compararlo con el sistema radical del pasto ryegrass.

Estas variables ambientales, que permite el crecimiento del cultivo, podrían generar condiciones durante el desarrollo de la planta que reducen su tasa de crecimiento (Gonçalves, Riberio, Reis, \& Cóser, 2018), al alcanzar periodos de nubosidad y precipitación que interfieren con la radiación que podría capturar el forraje. Dicha situación produce una reducción de la temperatura ambiental tanto en el día como en la noche, hasta el punto de inhibir el crecimiento del forraje, lo que reduce la productividad e incrementa los días de recuperación para obtener una mayor disponibilidad de forraje; sin dejar de lado el efecto físico del pisoteo de los semovientes sobre el cultivo y el suelo en condiciones con contenidos de humedad diferente (WingChing-Jones, Cabalceta-Aguilar, \& Alvarado-Hernández, 2009). Esta afirmación se fortalece al valorar la edad 
fenológica de los tres cultivos, donde por encima de los $2000 \mathrm{~m}$ s.n.m presentan una menor cantidad de hojas en desarrollo y se nota una tendencia a la disminución del número de hojas según aumenta la altitud. El uso de la edad fenológica para establecer los días de recuperación, permite optimizar la productividad de la pastura, siendo en el caso del pasto estrella (Villalobos \& Arce, 2013), kikuyo (Cascante, 2018) y ryegrass (Villalobos, 2006) de 6-8, 4,5 y 2,5-3,0, el número de hojas verdes apropiado para la cosecha respectivamente.

Al evaluar los aportes de proteína cruda, pared celular (FDN) y digestibilidad de la materia seca consumida, se observa que conforme aumenta la altitud, mayor es la concentración de proteína y materia seca digestible, lo que podría relacionarse a una mayor proporción entre el contenido celular y la pared celular de los forrajes evaluados. En estudios similares en Costa Rica, se determinaron valores de proteína de 19,79 a 20,79, 21,31 a 23,49 y de 24,32 a 36,30\% para los forrajes estrella (Salazar, 2007), kikuyo (Andrade, 2006) y ryegrass (Villalobos, 2006) cosechados a 28,28 y 40 días de recuperación respectivamente. Rangos que superan los valores determinados en este trabajo, diferencias que se podrían asociar al manejo de la pastura, a los programas de fertilización implementados y a las condiciones climáticas durante el periodo de evaluación (Redfearn, Venuto, Pitman, Alison, \& Ward, 2002). Además, estas diferencias fortalecen la importancia de establecer en los sistemas de producción, la práctica rutinaria de evaluación productiva y nutritiva de las pasturas, con la meta de satisfacer los requerimientos de los animales (NRC, 2001).

Al valorar las proporciones de las coberturas presentes, se nota como el tipo de crecimiento que presentan los tres forrajes evaluados, permiten la presencia de otras gramíneas, leguminosas y malezas. Como se comentó, el crecimiento estolonífero y rizomatoso permite una recuperación de la pastura que evita la proliferación de puntos de crecimientos de arvenses y leguminosas, como también, la competencia por otras gramíneas, caso contrario, el tipo cepa, característica del cultivo del ryegrass, donde por efecto del pisoteo y el pastoreo, estas unidades de crecimiento se debilitan y permite el ingreso del pasto kikuyo u otras gramíneas dentro del área de pastoreo de los sistemas de producción. Por otro lado, es importante considerar el manejo del pastoreo, donde la utilización del aparto por un periodo de medio día, genera una presión de pastoreo sobre el forraje, que en el momento de cambiar a los semovientes de aparto, el área recién pastoreada queda con un remanente aproximado al 55 al $68 \%$ de la biomasa inicial, material que cubre el suelo y evita la proliferación de otras coberturas (Villalobos, Arce, \& WingChing, 2013).

Las prácticas implementadas en el manejo de los cultivos en sistemas de producción especializada de leche en zona de altura como la suplementación en canoa, el pastoreo rotacional, presión de pastoreo no mayor a 12 horas continuas, uso de purines como enmiendas orgánicas (VanWieringen et al., 2005), aplicación de fertilizantes (N,P, K); y control cultural y químico de malezas, permiten mantener la cobertura principal del forraje, con la intención de garantizar un aporte de consumo de materia seca alto del forraje, debido a que es una fuente alimenticia de bajo costo (Villalobos, Arce, \& WingChing, 2013) en comparación a otras fuentes forrajeras, alimento balanceado y subproductos agrícolas utilizados en estos sistemas de producción.

\section{AGRADECIMIENTOS}

Agradecemos el apoyo brindado de la Vicerrectoría de Investigación de la Universidad de Costa Rica mediante el proyecto 739-B6-258 Estimación de biomasa con el plato medidor (Rising Platemeter) en pastos ryegrass, kikuyo y estrella africana. A los productores participantes, que de forma desinteresada nos permitieron trabajar en las áreas de pastoreo. 


\section{REFERENCIAS}

Andrade, M. (2006). Evaluación de técnicas de manejo para mejorar la utilización del pasto kikuyo (Pennisetum clandestinum_Hochst. Ex Chiov) en la producción de ganado lechero en Costa Rica (Tesis de Licenciatura). Universidad de Costa Rica, Costa Rica.

Association of Official Analytical Chemist (AOAC). (1991). Methods of analysis. Washington D.C., USA: AOAC, InC.

Belle, J. R., Marchi, S. R., Martins, D., Sousa, A. C., \& Pinheiro, G. H. R. (2018). Nutritional value of marandú palisade grass according to increasing coexistence periods with weed. Planta Daninha, 36, e018170348. DOI: 10.1590/S010083582018360100070

Cascante, S. (2018). Validación del sistema de pastoreo bajo el concepto de edad fenólogica del pasto Kikuyo (Kikuyuocloa clandestina) en la lechería La Guaria, de la Hacienda La Concordia, Alajuela, Costa Rica (Tesis de Licenciatura). Universidad de Costa Rica, Costa Rica.

Castillo, S. (2006). Uso de Metarhizium anisopliae para el control biológico del salivazo (Aeneolamia spp. y Prosapia spp.) en pastizales de Brachiaria decumbens en el Petén, Guatemala (Trabajo de maestría). CATIE, Costa Rica.

Cardamone, L., Cuatrín, A., Grunberg, K., \& Tomás, M. A. (2018). Variability for salt tolerance in a collection of Panicum coloratum var. Makarikariense during early growth stages. Tropical Grassland, 6(3), 134-147, DOI: 10.17138/TGFT(6)134-147

Conejo, J. (2017). Efecto de las condiciones ambientales sobre la producción láctea de dos hatos de ganado Jersey en dos pisos altitudinales de la provincia de Cartago (Tesis de Licenciatura). Universidad de Costa Rica, Costa Rica.

Galon, L., Santin, C. O., Andres, A., Basso, F. J. M., Nonemacher, F., Agazzi, L. R., ... Fernandez, F. F. (2018). Competitive interaction between sweet sorghum with weed. Planta Daninha, 36, e018173689, DOI: 10.1590/S010083582018360100053

Garzón, E. F. \& Mora, J. (2014). Análisis multicriterio del estado de las pasturas de la Hacienda Ganadera García abajo en Corinto (Cuaca, Colombia). Revista de la Facultad de Medicina Veterinaria y de Zootecnia, 61(1), 64-82. DOI: 10.15446/rfmvz.v61n1.44182

Gonçalves, M., Ribeiro, W., Reis, E., \& Cóser A. (2018). Bromatología de gramíneas tropicais sob diferentes tensões de água no solo em ambiente protegido. Nativa, 6(4), 415-421. DOI: 10.31413/nativa.v6i4.5588

Hargraves, J. N. G., \& Kerr J. D. (1978). Botanal: a comprehensive sampling and computing procedure for estimating pasture yield and composition. II. Computational package (Technical Memorandum №. 9). Division of Tropical Crops and Pastures, Tropical Agronomy, CSIRO, Australia.

Harker, K., Baron, V., Chanasyk, D., Naeth, M., \& Stevenson, F. (2000) Grazing intensity effects on weed populations in annual and perennial pasture systems. Weed Science, 48(2), 231-238. DOI: 10.1614/00431745(2000)048[0231:GIEOWP]2.0.CO;2

INEC (Instituto Nacional de Estadísticas y Censos). (2015). VI Censo Nacional Agropecuario: Resultados generales. Instituto Nacional de Estadística y Censos, San José, CRC. Recuperado de http://www.mag.go.cr/bibliotecavirtual/U4010581.pdf

Jiménez, O. M. M., Granados, L., Oliva, J., Quiroz, J., \& Barrón, M. (2010). Calidad nutritiva de Brachiaria humidicola con fertilización orgánica e inorgánica en suelos ácidos. Archivos de Zootecnia, 59(228), 561-570. DOI: 10.21071/az.v59i228.4711

NRC (National Research Council). (2001). Nutrient requirements of dairy cattle (7th Ed.). National Academy Press, Washington DC., USA. 
Núñez, L. (2017). Identificación de nematodos fitoparásitos asociados a las principales malezas en fincas productoras de papa (Solanum tuberosum L.) en la provincia de Cartago (Tesis de Licenciatura). Universidad de Costa Rica, Costa Rica.

Redfearn, D., Venuto, B., Pitman, D., Alison, M., \& Ward, J. (2002). Cultivar and environment effects on anual ryegrass forage yield, yield distribution and nutritive value. Crop Science, 42, 2049-2054. DOI: 10.2135/cropsci2002.2049

Salazar, S. (2007). Disponibilidad de biomasa y valor nutricional del pasto estrella africana (Cynodon nlemfuensis) en el distrito de Quesada, Cantón de San Carlos. (Tesis de Licenciatura). Universidad de Costa Rica, San José, Costa Rica.

SAS. (2011). The SAS system for Windows No. 9.3. SAS Inst., Cary, NC, USA.

VanWieringen, L., Harrison, J., Nennich, T., Davidson, D., Morgan, L., Chen, S., ... Hoisington, F. (2005). Manure management effects on grass production, nutritive content and soil nitrogen for a grass silage-based dairy farm. Journal Environmental Quality, 34, 164-173.

Villalobos, L. (2006). Disponibilidad y valor nutricional del pasto ryegrass perenne tetraploide (Lolium perenne) en las zonas altas de Costa Rica (Tesis de Licenciatura). Universidad de Costa Rica, Costa Rica.

Villalobos, L., \& Arce, J. (2013). Evaluación agronómica y nutricional del pasto Estrella africana (Cynodon nlemfuensis) en la zona de Monteverde, Puntarenas, Costa Rica. I. Disponibilidad de biomasa y fenología. Agronomía Costarricense, 37, 91-101.

Villalobos, L., Arce, J., \& WingChing, R. (2013). Producción de biomasa y costos de producción de pastos Estrella africana (Cynodon nlemfuensis), Kikuyo (Kikuyocloa clandestina) y Ryegrass perenne (Lolium perenne) en lecherías de Costa Rica. Agronomía Costarricense, 37(2), 91-103.

WingChing-Jones, R., Cabalceta-Aguilar, G., \& Alvarado-Hernández, A. (2009). Impacto del pastoreo con ganado Holstein y Jersey sobre la densidad aparente de un andisol. Agronomía Mesoamericana, 20(2), 369-377. DOI: 10.15517/am.v20i2.4953

Yuan, Z., Zheng, X., Zhao, Y., Liu, Y., Zhou, S., Wei, C., ... Shao, H. (2018). Phytotoxic compounds isolated from leaves of the invasive weed Xanthium spinosum. Molecules, 23(11), 2840. DOI: 10.3390/molecules23112840 